\title{
SINGULARITIES OF CURVES OF GIVEN ORDER *
}

BY T. R. HOLLCROFT

1. Introduction. The following problems concerning plane curves are suggested by the theory of surfaces and space curves.

I. To find the greatest number of cusps that may be added to the singularities of a plane curve of order $n$ with $\kappa_{1}$ cusps and $\delta_{1}$ nodes.

II. To find the greatest number of nodes that may be added to the singularities of a plane curve of order $n$ with $\kappa_{1}$ cusps and $\delta_{1}$ nodes.

III. To find the greatest number of cusps that may occur among the remaining singularities of a plane curve of order $n$ and genus $p$ with $\kappa_{1}$ cusps and $\delta_{1}$ nodes.

IV. To find the least order $n$ of a plane curve that can possess a given number $\kappa_{1}$ of cusps and $\delta_{1}$ of nodes.

These problems are all closely related to the question of the existence of plane curves with assigned singularities, in which the principal problem consists of finding the maximum number of cusps of a curve of given order and genus. This problem has been solved by Lefschetz, $\uparrow$ who also mentions the first two of the above problems, but suggests only graphical solutions for them. The existence of curves with cusps up to and including the maximum has been shown by both Lefschetz and Coolidge $\ddagger$ for $p \leqq p_{0}$, where $p_{0}$ is the genus associated with a given $n$ on the assumption that the bitangents and inflections are both as near zero as possible.

As a notation for the number of singularities of any algebraic plane curve of order $n$, class $m$, and genus $p$, we shall say that

* Presented to the Society, April 28, 1923.

$\dagger \mathrm{S}$. Lefschetz, On the existence of loci with given singularities, TransACTIONS OF THIS SocIETY, vol. 14 (1913), pp. 23-41.

$\ddagger J$. L. Coolidge, On the existence of curves with assigned singularities, this Bulletin, vol. 28 (1922), pp. 451-455. 
the curve has $\kappa$ cusps, $\delta$ nodes, $\iota$ inflections, and $\tau$ bitangents. We shall have occasion to use the following formulas, the first three of which are Plückerian:

$$
\begin{aligned}
\text { (1) } \quad m & =n(n-1)-2 \delta-3 \kappa, \\
\text { (2) } \quad \iota & =3 n(n-2)-6 \delta-8 \kappa, \\
\text { (3) } 2 \tau & =2 \delta+(m-n)(m+n-9), \\
\text { (4) } \quad F & =\frac{1}{2} n(n+3)-\delta-2 \kappa-8=3 n+p-9-\kappa,
\end{aligned}
$$

$$
p \geqq 2 .
$$

The last formula, due to Professor Lefschetz, ${ }^{*}$ gives the number of independent absolute invariants necessary to determine a curve of order $n$ and genus $p \geqq 2$ with $\delta$ nodes and $\kappa$ cusps.

The conditions for the maximum number of cusps of a curve of given order and genus are obtained by Lefschetz for certain limits of $n$ in terms of a given $p$. If we restate them for certain limits of $p$ in terms of a given $n$, we find that for $p \leqq p_{0}$, where $p_{0}$ is defined by

$$
\left[p_{0}\right] \dagger \leqq \frac{1}{2}(n+2-\sqrt{4 n+13}),
$$

$\kappa$ is a maximum when $\iota$ is a minimum; for $p_{0}<p \leqq p_{1}$, where

$$
\left[p_{1}\right] \leqq \frac{1}{2}(2 n-1+\sqrt{16 n-23}),
$$

$\boldsymbol{\kappa}$ is a maximum when $\tau$ is a minimum; and for

$$
p_{1} \leqq p \leqq \frac{1}{4}(n-4)(n-5) \quad \text { and } \quad n>13 \text {, }
$$

$\kappa$ is limited by the number of independent absolute invariants determining the curve.

In all four problems, let $\kappa_{1}$ and $\delta_{1}$ represent, respectively, the number of cusps and nodes already possessed by the curve of order $n$ and let $\kappa_{m}$ represent the maximum number of cusps that a curve of order $n$ with $\delta_{1}$ nodes may have. The solutions will now be given.

* S. Lefschetz, loc. cit., p. 29.

$\dagger$ In this paper, the symbol $[x]$ followed by $\leqq(\geqq)$ means the largest (one greater than the largest if not equal) integer contained in the expression on the right. The interpretation not in parentheses is the usual one. 
2. Problem I. From formula (2) we obtain, by means of the condition $\iota \geqq 0$,

$$
\left[\kappa_{m}\right] \leqq \frac{3}{8}\left(n^{2}-2 n-2 \delta_{1}\right) \text {. }
$$

Substituting the value of $m$ from (1) in (3), letting $\tau \geqq 0$, and solving for $\kappa$, we obtain

(6) $\left[\kappa_{m}\right] \geqq \frac{1}{6}\left[2 n(n-1)-4 \delta_{1}-9-\sqrt{4 n(n-9)-8 \delta_{1}+81}\right]$. If we let $F \geqq 0$ in (4), we find

$$
\left[\kappa_{m}\right] \leqq \frac{1}{4}\left[n(n+3)-2\left(\delta_{1}+8\right)\right] \text {. }
$$

For a given $n$ and $\delta_{1}$, the one of these three formulas that gives the least value of $\kappa_{m}$ determines the maximum number of cusps that curve can have.

It is now necessary to find the limits of $\delta_{1}$ for which each of these formulas applies. If

$$
\delta_{1} \geqq \frac{1}{2}(n-2)(n-4),
$$

the curve may be rational and have all the rest of its double points cusps. The limit (5) holds for $0 \leqq p \leqq p_{0}$ and for these curves

$$
[\delta] \leqq \frac{1}{2}(n-2)(n-4)-4 p .
$$

Replacing $\delta$ by $\delta_{1}$, and eliminating $p$ from this formula and the upper limit for $p$, we obtain

$$
\delta_{1} \geqq \frac{1}{2} n(n-10)+2 \sqrt{4 n+13} .
$$

To find the lower limit of $\delta_{1}$ for formula (6) when $n>13$, eliminate $p$ from the inequality for $p_{1}$ and the formula for $\delta$, when $p \geqq p_{1}$,

This gives

$$
\delta=\frac{1}{2}(n-4)(n-5)-2 p .
$$

$$
\delta_{1} \geqq \frac{1}{2}(n-2)(n-11)-\sqrt{16 n-23} .
$$

The complete solution may now be stated as follows: If If

$$
\begin{aligned}
& \delta_{1} \geqq \frac{1}{2}(n-2)(n-4), \\
& \kappa_{m}=\frac{1}{2}(n-1)(n-2)-\delta_{1} . \\
& \frac{1}{2}(n-2)(n-4) \geqq \delta_{1} \geqq \frac{1}{2} n(n-10)+2 \sqrt{4 n+13}, \\
& {\left[\kappa_{m}\right] \leqq \frac{3}{8}\left(n^{2}-2 n-2 \delta_{1}\right) .}
\end{aligned}
$$


If If

$$
\begin{aligned}
& \frac{1}{2} n(n-10)+2 \sqrt{4 n+13}>\delta_{1}\left\{\begin{array}{c}
\geqq 0 \quad \text { for } n \leqq 13, \\
\geqq \frac{1}{2}(n-2)(n-11) \\
-\sqrt{16 n-23} \\
\text { for } n>13,
\end{array}\right. \\
& {\left[\kappa_{m}\right] \geqq \frac{1}{6}\left[2 n(n-1)-4 \delta_{1}-9-\sqrt{4 n(n-9)-8 \delta_{1}+81}\right] .} \\
& \frac{1}{2}(n-2)(n-11)-\sqrt{16 n-23} \geqq \delta_{1} \geqq 0 \text { for } n>13, \\
& {\left[\kappa_{m}\right] \leqq \frac{1}{4}\left[n(n+3)-2\left(\delta_{1}+8\right)\right] .}
\end{aligned}
$$

In any of these cases, the number of cusps that may be added is $\kappa_{m}-\kappa_{1}$, and there may be one additional node if $\kappa_{m}$ is not diminished by substituting $\delta_{1}+1$ for $\delta_{1}$ in the limit for $\kappa_{m}$.

3. Problem II. Let $\delta_{m}$ denote the greatest number of nodes (including $\delta_{1}$ ) that the curve of order $n$ with $\kappa_{1}$ cusps can have. Solve the three formulas (5), (6), (7) for $\delta$ in terms of $n$ and $\kappa_{1}$ and find the limits of $\kappa_{1}$ for which they hold in the same way that the limits for $\delta_{1}$ were found in the preceding problem In this case there can be no additional cusps. The results are as follows: If $\kappa_{1} \leqq \frac{3}{2}(n-2)$, we have

$$
\delta_{m}=\frac{1}{2}(n-1)(n-2)-\kappa_{1} .
$$

If

$$
\begin{aligned}
& \frac{3}{2}(n-2) \leqq \kappa_{1} \leqq \frac{3}{2}(2 n-\sqrt{4 n+13}), \\
& {\left[\delta_{m}\right] \leqq \frac{1}{6}\left[3 n(n-2)-8 \kappa_{1}\right] .}
\end{aligned}
$$

If

$$
\begin{aligned}
& \frac{3}{2}(2 n-\sqrt{4 n+13})<\kappa_{1}\left\{\begin{array}{c}
\leqq \frac{1}{3} n(n-2) \quad \text { for } \quad n \leqq 13, \\
\leqq \frac{1}{2}(8 n-19+\sqrt{16 n-23}) \\
\text { for } \quad n>13
\end{array}\right. \\
& {\left[\delta_{m}\right] \leqq \frac{1}{2}\left(n^{2}-n-3 \kappa_{1}-5-\sqrt{\left.3 \kappa_{1}-8 n+25\right)} .\right.}
\end{aligned}
$$

If

$$
\begin{aligned}
& \frac{1}{2}(8 n-19+\sqrt{16 n-23}) \leqq \kappa_{1} \leqq \frac{1}{4}\left(n^{2}+3 n-16\right) \\
& {\left[\delta_{m}\right] \leqq \frac{1}{2}\left(n^{2}+3 n-16-4 \kappa_{1}\right) .}
\end{aligned}
$$

In any case, the number of nodes that may be added is given by the difference $\delta_{m}-\delta_{1}$. 
4. Problem III. In this problem $p, \kappa_{1}$, and $\delta_{1}$ must satisfy the inequality $\frac{1}{2}(n-1)(n-2)-\kappa_{1}-\delta_{1} \geqq p \geqq \frac{1}{2}(n-1)(n-2)-\kappa_{1}-\delta_{m}$.

The problem is solved by noting that the maximum number of cusps depends only on $n$ and $p$ when

$$
\delta_{1} \leqq \frac{1}{2}(n-1)(n-2)-\kappa_{m}-p,
$$

and when $\delta_{1}$ equals or exceeds this limit, all the remaining double points may be cusps. The following formulas result: If

and

$$
0 \leqq p \leqq \frac{1}{2}(n+2-\sqrt{4 n+13}),
$$

$$
\begin{aligned}
& \delta_{1} \leqq \frac{1}{2}(n-2)(n-4)-4 p, \\
& {\left[\kappa_{m}\right] \leqq \frac{3}{2}(n+2 p-2) .}
\end{aligned}
$$

If

$$
\frac{1}{2}(n+2-\sqrt{4 n+13})<p \leqq \frac{1}{2}(2 n-1+\sqrt{16 n-23})
$$

and

$$
\begin{aligned}
& \delta_{1} \leqq \frac{1}{2}\left(n^{2}-7 n+13-6 p+\sqrt{24 p-8 n+25}\right) \\
& {\left[\kappa_{m}\right] \leqq 2(n+p)-\frac{1}{2}(11+\sqrt{24 p-8 n+25}) .}
\end{aligned}
$$

If

$$
\frac{1}{2}(2 n-1+\sqrt{16 n-23}) \leqq p \leqq \frac{1}{4}(n-4)(n-5)
$$

and If

$$
\begin{aligned}
& \delta_{1} \leqq \frac{1}{2}(n-4)(n-5)-2 p, \\
& \kappa_{m}=3 n+p-9 .
\end{aligned}
$$

$$
p>\frac{1}{4}(n-4)(n-5),
$$

or if $\delta_{1}$ equals or exceeds any of the above limits,

$$
\kappa_{m}=\frac{1}{2}(n-1)(n-2)-\delta_{1}-p .
$$

In any case, the number of cusps that may be added is $\kappa_{m}-\kappa_{1}$, and the additional nodes, if any, that belong to the curve, are given by the formula

$$
\delta=\frac{1}{2}(n-1)(n-2)-\kappa_{m}-\delta_{1}-p .
$$


The solution of the problem, to find the maximum number of nodes that may occur among the remaining singularities of a curve of order $n$ and genus $p$ with $\kappa_{1}$ cusps and $\delta_{1}$ nodes, is evidently always

$$
\delta_{m}-\delta_{1}=\frac{1}{2}(n-1)(n-2)-\kappa_{1}-\delta_{1}-p,
$$

since $p$ cannot be less than the genus of a curve with $\kappa_{1}$ cusps and the maximum number of nodes.

5. Problem IV. For a rational curve we have

$$
\begin{aligned}
& \delta_{1} \geqq \frac{1}{2}(n-2)(n-4), \\
& \kappa_{1}=\frac{1}{2}(n-1)(n-2)-\delta_{1} .
\end{aligned}
$$

Solving (9) for $n$, we have

$$
n=\frac{1}{2}\left[3+\sqrt{8\left(\kappa_{1}+\delta_{1}\right)+1}\right] .
$$

Substituting this value of $n$ in (8), and solving for $\delta_{1}$, we obtain

$$
\delta_{1} \geqq \frac{2}{9} \kappa_{1}\left(\kappa_{1}-3\right),
$$

which gives the relation between $\delta_{1}$ and $\kappa_{1}$ for which the above formula holds.

When $\delta_{1}<2 \kappa_{1}\left(\kappa_{1}-3\right) / 9$ the curve cannot be rational and we must consider three cases, $0 \leqq p \leqq p_{0}, p_{0}<p \leqq p_{1}$ and $p \geqq p_{1}$. For $p \leqq p_{0}$ the maximum number of cusps occurs when the number of inflections is a minimum. Solving the inequality resulting from equation (2) for $n$, we find

$$
[n] \geqq \frac{1}{3}\left[3+\sqrt{3\left(3+6 \delta_{1}+8 \kappa_{1}\right)}\right] .
$$

Eliminating $p$ and $n$ from the inequality defining $p_{0}$ and the two inequalities

$$
\begin{aligned}
& {\left[\delta_{1}\right] \geqq \frac{1}{2}(n-2)(n-4)-4 p,} \\
& {\left[\kappa_{1}\right] \leqq \frac{3}{2}(n+2 p-2),}
\end{aligned}
$$

which give the nodes and the maximum number of cusps for $p \leqq p_{0}$, we obtain

$$
\delta_{1} \geqq \frac{1}{18}\left[\kappa_{1}\left(\kappa_{1}-21\right)+2 \kappa_{1} \sqrt{3\left(\kappa_{1}+3\right)}\right] .
$$

If $p_{0}<p \leqq p_{1}$, the maximum number of cusps occurs when 
the number of double tangents is a minimum. If we attempt to solve the resulting inequalities in $\kappa_{1}, \delta_{1}$ and $n$ for $n$, we are led to the quartic equation

$$
\begin{aligned}
n^{4}-2 n^{3}-\left[2 \left(3 \kappa_{1}+\right.\right. & \left.\left.2 \delta_{1}\right)+9\right] n^{2}+2\left(3 \kappa_{1}+2 \delta_{1}+9\right) n \\
& +\left(3 \kappa_{1}+2 \delta_{1}\right)\left(3 \kappa_{1}+2 \delta_{1}+9\right)+2 \delta_{1}=0 .
\end{aligned}
$$

A general solution of this equation is too involved to be of use as a formula, but the value of $n$ for given values of $\kappa_{1}$ and $\delta_{1}$ can be found as the least positive real root of this quartic when that root is an integer, or the integer next larger when the least positive root is irrational.

If

$$
\frac{1}{18}\left[\kappa_{1}\left(\kappa_{1}-21\right)+2 \kappa_{1} \sqrt{3\left(\kappa_{1}+3\right)}\right]>\delta_{1} \geqq 0,
$$

and $\kappa_{1} \leqq 50$ the foregoing formula applies. If $\kappa_{1}>50$, however, the lower limit for $\delta_{1}$, for which the foregoing formula applies, is not zero, but we cannot find it in general, since it involves the solution of two quartic equations.

If $\kappa_{1}>50$, and $\delta_{1}$ is less than this lower limit, $p>p_{1}$, and the least value of $n$ is obtained by eliminating $p$ from the two formulas

$$
\begin{aligned}
\kappa_{1} & =3 n+p-9, \\
p & =\frac{1}{2}(n-1)(n-2)-\delta_{1}-\kappa_{1} .
\end{aligned}
$$

Solving these for $n$, we have

$$
[n] \geqq \frac{1}{2}\left(\sqrt{16 \kappa_{1}+8 \delta_{1}+73}-3\right) .
$$

The inequality sign is introduced in the result because $\delta_{1}$ may not have the value that will satisfy the equality.

In general, when it is desired to find the least order $n$ of a curve that can possess a given number $\kappa_{1}$ of cusps and $\delta_{1}$ of nodes, we may proceed as follows. If

If

$$
\begin{aligned}
& \delta_{1} \geqq \frac{2}{9} \kappa_{1}\left(\kappa_{1}-3\right), \\
& {[n]=\frac{1}{2}\left[3+\sqrt{8\left(\kappa_{1}+\delta_{1}\right)+1}\right] .}
\end{aligned}
$$

$$
\begin{aligned}
& \frac{2}{9} \kappa_{1}\left(\kappa_{1}-3\right)>\delta_{1} \geqq \frac{1}{18}\left[\kappa_{1}\left(\kappa_{1}-21\right)+2 \kappa_{1} \sqrt{3\left(\kappa_{1}+3\right)}\right], \\
& {[n] \geqq \frac{1}{3}\left[3+\sqrt{3\left(3+6 \delta_{1}+8 \kappa_{1}\right)}\right] .}
\end{aligned}
$$

If

$$
\frac{1}{18}\left[\kappa_{1}\left(\kappa_{1}-21\right)+2 \kappa_{1} \sqrt{3\left(\kappa_{1}+3\right)}\right]>\delta_{1} \geqq 0 \text { and } \kappa_{1} \leqq 50 \text {, }
$$


$n$ is given as the least positive real root, if that is an integer, or the integer next larger than the least positive irrational root of the quartic

$$
\begin{aligned}
n^{4}-2 n^{3}-\left[2 \left(3 \kappa_{1}+\right.\right. & \left.\left.2 \delta_{1}\right)+9\right] n^{2}+2\left(3 \kappa_{1}+2 \delta_{1}+9\right) n \\
& +\left(3 \kappa_{1}+2 \delta_{1}\right)\left(3 \kappa_{1}+2 \delta_{1}+9\right)+2 \delta_{1}=0 .
\end{aligned}
$$

If $\kappa_{1}>50$, determine $n$ by the inequality

$$
[n] \geqq \frac{1}{2}\left(\sqrt{16 \kappa_{1}+8 \delta_{1}+73}-3\right) .
$$

If $p \geqq p_{1}$ for this value of $n$ and the given values of $\kappa_{1}$ and $\delta_{1}$, this is the correct value for $n$; but if $p<p_{1}$, this formula does not apply, and $n$ must be determined from the quartic equation as above.

When $\delta_{1}=0$, the solution is more simple. For $n \leqq 13$, the maximum number of cusps is given by $[k] \leqq \frac{1}{3} n(n-2)$. Hence, solving this inequality for $n$, we have

For $n>13$,

$$
[n] \geqq 1+\sqrt{3 \kappa_{1}+1} \quad \text { for } \quad \kappa_{1} \leqq 50 .
$$

$$
[\kappa] \leqq \frac{1}{4} n(n+3)-4 .
$$

Solving for $n$ we obtain

$$
[n] \geqq \frac{1}{2}\left(\sqrt{16 \kappa_{1}+73}-3\right) \quad \text { for } \quad \kappa_{1}>50 .
$$

In the foregoing results, nothing has been said as to the relative positions of the double points. None of the curves can have more double points than has been assigned them, but in certain cases some of these double points may unite to form points of higher multiplicity. When the genus does not change, one additional independent absolute invariant is imposed on the curve for each increase in the multiplicity of a point by unity. For these curves, the only restriction on the coincidence of double points is that the total increase in multiplicity shall not exceed $3 n+p-9-\kappa$. For $p<p_{1}$, this allows a certain number of coincidences, but for $p \geqq p_{1}$, all the double points assigned must be distinct for a proper curve.

Wells College 